\title{
Demystifying Commercial Video Conferencing Applications
}

\author{
Insoo Lee \\ University of Colorado Boulder \\ Insoo.Lee@colorado.edu
}

\author{
Jinsung Lee \\ University of Colorado Boulder \\ Jinsung.Lee@colorado.edu
}

\author{
Kyunghan Lee \\ Seoul National University \\ kyunghanlee@snu.ac.kr
}

\author{
Dirk Grunwald \\ University of Colorado Boulder \\ Dirk.Grunwald@colorado.edu
}

\author{
Sangtae Ha \\ University of Colorado Boulder \\ Sangtae.Ha@colorado.edu
}

\begin{abstract}
Video conferencing applications have seen explosive growth both in the number of available applications and their use. However, there have been few studies on the detailed analysis of video conferencing applications with respect to network dynamics, yet understanding these dynamics is essential for network design and improving these applications. In this paper, we carry out an in-depth measurement and modeling study on the rate control algorithms used in six popular commercial video conferencing applications. Based on macroscopic behaviors commonly observed across these applications in our extensive measurements, we construct a unified architecture to model the rate control mechanisms of individual applications. We then reconstruct each application's rate control by inferring key parameters that closely follow its rate control and quality adaptation behaviors. To our knowledge, this is the first work that reverse-engineers rate control algorithms of popular video conferencing applications, which are often unknown or hidden as they are proprietary software. We confirm our analysis and models using an end-to-end testbed that can capture the dynamics of each application under a variety of network conditions. We also show how we can use these models to gain insights into the particular behaviors of an application in two practical scenarios.
\end{abstract}

\section{CCS CONCEPTS}

\section{- Information systems $\rightarrow$ Multimedia streaming.}

\section{KEYWORDS}

Video conferencing; live video; rate control; QoE; measurement

\section{ACM Reference Format:}

Insoo Lee, Jinsung Lee, Kyunghan Lee, Dirk Grunwald, and Sangtae Ha. 2021. Demystifying Commercial Video Conferencing Applications. In Proceedings of the 29th ACM International Conference on Multimedia (MM '21), October 20-24, 2021, Virtual Event, China. ACM, New York, NY, USA, 9 pages. https: //doi.org/10.1145/3474085.3475523

\section{INTRODUCTION}

The demand for video conferencing has surged exponentially with the result of today's changing nature of work. Even before the

This work is licensed under a Creative Commons Attribution-NonCommercialNoDerivs International 4.0 License.

MM '21, October 20-24, 2021, Virtual Event, China.

(c) 2021 Copyright held by the owner/author(s).

ACM ISBN 978-1-4503-8651-7/21/10.

https://doi.org/10.1145/3474085.3475523 pandemic arrived, the need was already on the rise at an accelerated pace [30]. Many popular video conferencing applications [5, 28, 40] support collaboration features such as screen sharing, whiteboard, live chat, and annotation to make virtual meetings as productive as in-person meetings. While it may be harder to define, the most important feature of these applications is providing reliable video communication among participants.

However, users often face poor video quality and latency issues even though the network exhibits no apparent problems. To tackle this situation, they attempt to reconnect or switch to a different video conferencing application to see if this could improve the current situation, without knowing how this new application will adapt to poor network conditions compared to the previous application.

This is why the design of an efficient rate control algorithm for modern video conferencing applications has been an important research topic. The rate control algorithm for the live video streaming typically has two main functions: (1) it estimates time-varying network conditions such as bandwidth, packet loss, and end-to-end latency; and (2) it provides an estimated sending rate for the video encoder to adjust the source rate (i.e., encoding rate) for low-latency streaming. Unfortunately, however, most commercial applications' rate control algorithms are largely unknown due to their closed source policy to protect their intellectual properties, except for Google's WebRTC [18], whose default rate control algorithm is the open-sourced Google Congestion Control (GCC) [4, 21].

In this paper, we analyze WebRTC and six other popular video conferencing applications according to Gartner's market analysis [14]: Bluejeans, Google Duo, Gotomeeting, Skype, Cisco Webex, and Zoom. From our pilot measurement (detailed in §2), we observe that no single video application achieves consistently high performance across different network scenarios. We also find that these applications exhibit distinct responses against network dynamics (i.e., loss, delay, and bandwidth changes). This quantitative analysis motivates our research with the following question: Can we infer rate control and video quality adaptation mechanisms of commercial video conferencing applications?

Since it is impossible to infer the hidden behaviors of each application's rate control algorithm solely from passive measurements, we take two approaches. First, we estimate the sensitivity parameters for packet loss and latency and video quality adaptation of each application by varying the network bandwidth, latency, and packet loss rates in a binary search manner, which is inspired from previous evaluation studies [36, 38]. In particular, we design a small set of artificial network scenarios that can capture each application's rate and video quality control. We also develop an accurate estimation method to obtain the resolution and frame rate of video streaming 

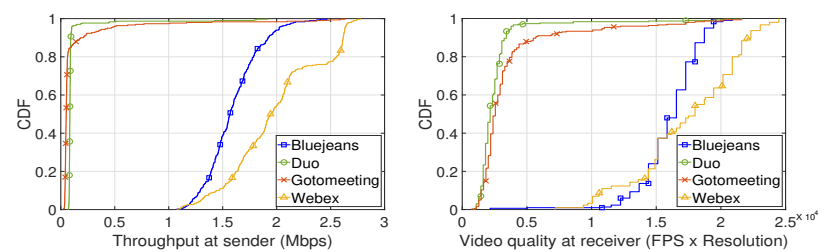

Figure 1: The CDF of throughput and video quality for applications using cloud servers under delay variation.

since most applications hide this information from users. Second, once we derive the rate control parameters for a given application, we use an open-source WebRTC as a baseline architecture to model and validate each application's rate control mechanism.

In summary, we have the following contributions:

- We conduct an in-depth comparison of six commercial video conferencing applications (§2). Our key observations provide guidelines to infer the proprietary rate control algorithms employed by different applications.

- To our knowledge, this is the first work that derives the unknown rate control algorithms used by the commercial video conferencing applications ( $\$ 3$ and $\S 4)$. We apply the inferred parameters on WebRTC's rate control framework to validate our derived model for each application that matches its rate control behavior.

- We construct the models for the rate control in the transport layer and the QoE (Quality of Experience)-related metrics in the application layer for six video conferencing applications $(\S 4)$. We confirm that our inferred models follow their dynamics across various network scenarios (§5), providing a systematic way to understand their rate and quality adaptation behaviors.

- We analyze several practical scenarios with inferred models (§6). Our model-driven analysis justifies why different applications show contrasting behaviors when competing with other flows.

- Our analysis using sensitivity parameters provides a scientific way to explain and compare the design choices of each video conference application. It will open up new research, such as 1) designing a better rate and QoE control algorithm and 2) traffic engineering and capacity planning for these applications.

\section{MEASUREMENT STUDY}

We present the essential characteristics of six video conferencing applications. Using our in-lab network testbed (detailed in §3.1), we carry out extensive measurements to infer their operating ranges and understand the impact of various network factors on their performance. This will be the foundation of our design to infer each application's rate control algorithm in the following sections. The measurement dataset and tools are available at [7].

\subsection{Basic Characteristics}

As shown in Table 1, we observe that each application reveals a different maximum video bitrate and frame rate in the network with sufficient bandwidth. As the emulated network does not impose any constraints on packet loss or bandwidth throttling, we can obtain the maximum video bitrate, which is nearly the same as the sending rate except for the header overhead. To stream a HD video $(1280 \times 720$ resolution) at the frame rate of more than $25 \mathrm{FPS}$, there must be at least $1.8 \mathrm{Mbps}$ bandwidth over the end-to-end path. Furthermore, each application has a difference in serving multiple participants. Skype and Zoom prefer a direct, peer-topeer connection for the call between the two peers while the other applications use cloud servers (as relay) even for two-party calls.

More importantly, we observe that the sender's rate control algorithm primarily affects the receiver's video rate and quality for both peer-to-peer and cloud-assisted connection types. As shown in Figure 1, the four applications using cloud-assisted connections show a strong positive relationship between the sender's rate and the receiver's rate (or quality). This makes sense since the cloud server will deliver the receiver as much as it receives from the sender (not more than that), which is not different from the case where the client receives what the sender sends for peer-to-peer, two-party calls. Thus, we know that the sender's rate control behaviors impact the user experience of all video conferencing applications.

\subsection{Impact of Network Parameters}

We perform extensive measurements by varying network parameters such as bandwidth, delay, and packet loss to induce how their proprietary rate control algorithms react to different network conditions. We use an end-to-end emulated network testbed (Figure 2) detailed in $\S 3.1$ and report only key observations.

Figure 3 compares the performance of average normalized throughput and one-way streaming latency achieved by the six applications and WebRTC under three different network conditions: (i) packet loss rate from 0 to $20 \%$, (ii) additional one-way delay from 0 to $375 \mathrm{~ms}$, and (iii) bandwidth from $500 \mathrm{Kbps}$ to $5 \mathrm{Mbps}$. The upper left direction indicates better performance because a video stream quality improves with high throughput while being resistant to increasing network latency. The overall results indicate that the adaptation behavior of each application is different from the other, and no single application behaves superior in all of these scenarios.

We also report how each application changes its rate control behavior under different network conditions below.

Impact of available bandwidth. We check each application's adaptation behavior while varying the bandwidth from $200 \mathrm{Kbps}$ to $5 \mathrm{Mbps}$ in $100 \mathrm{Kbps}$ steps. We find that all video conferencing applications keep track of the available bandwidth with different levels of a margin such that they achieve maximum attainable bitrate while not causing any significant congestion.

Impact of propagation delay. We added the additional propagation delay from $0 \mathrm{~ms}$ to $400 \mathrm{~ms}$ while fixing the available bandwidth and packet loss rate at $10 \mathrm{Mbps}$ and $0 \%$, respectively. Our measurement results show that all applications maintain their bitrate while video streaming delay increases linearly, as the propagation delay increases, leading rate insensitivity to the fixed propagation delay. Impact of delay variance. We insert a sudden additional delay to the applications to see if their rate controls are different from the fixed propagation delay case above. We observe that all applications temporarily lower their bit rate due to the extra delay but recover shortly. We conjecture that delay-based rate adaptation mechanisms in the video conferencing applications use the delay variation rather than its fixed value. This design choice is similar to the default GCC algorithm used in WebRTC that utilizes the one-way delay variation named delay gradient to infer congestion [4].

Impact of packet loss rates. We vary the packet loss rates, ranging from $0 \%$ to $10 \%$, while fixing the bandwidth and propagation 


\begin{tabular}{|c|c|c|c|c|c|}
\hline Application & $\begin{array}{c}\text { Connection } \\
\text { type }\end{array}$ & $\begin{array}{c}\text { Max. } \\
\text { video bitrate }\end{array}$ & $\begin{array}{c}\text { Max. } \\
\text { frame rate }\end{array}$ & $\begin{array}{c}\text { Observed resolution } \\
\text { (height pixels) }\end{array}$ & $\begin{array}{c}\text { Recommended } \\
\text { bandwidth }\end{array}$ \\
\hline \hline Bluejeans & Cloud Server & $2.0 \mathrm{Mbps}$ & $25 \mathrm{FPS}$ & $180 \mathrm{p}, 288 \mathrm{p}, 360 \mathrm{p}, 480 \mathrm{p}, 720 \mathrm{p}$ & $1.5 \mathrm{Mbps}$ \\
\hline Google Duo & Cloud Server & $1.8 \mathrm{Mbps}$ & $30 \mathrm{FPS}$ & $180 \mathrm{p}, 270 \mathrm{p}, 360 \mathrm{p}, 450 \mathrm{p}, 540 \mathrm{p}, 720 \mathrm{p}$ & $1.0 \mathrm{Mbps}$ \\
\hline Gotomeeting & Cloud Server & $2.6 \mathrm{Mbps}$ & $30 \mathrm{FPS}$ & $180 \mathrm{p}, 270 \mathrm{p}, 360 \mathrm{p}, 450 \mathrm{p}, 540 \mathrm{p}, 720 \mathrm{p}$ & $1.0 \mathrm{Mbps}$ \\
\hline Skype & Direct & $2.4 \mathrm{Mbps}$ & $28 \mathrm{FPS}$ & $180 \mathrm{p}, 270 \mathrm{p}, 360 \mathrm{p}, 450 \mathrm{p}, 480 \mathrm{p}, 540 \mathrm{p}, 720 \mathrm{p}$ & $1.5 \mathrm{Mbps}$ \\
\hline Cisco Webex & Cloud Server & $1.8 \mathrm{Mbps}$ & $26 \mathrm{FPS}$ & $90 \mathrm{p}, 180 \mathrm{p}, 360 \mathrm{p}, 432 \mathrm{p}, 576 \mathrm{p}, 648 \mathrm{p}, 720 \mathrm{p}$ & $1.75 \mathrm{Mbps}$ \\
\hline Zoom & Direct & $3.3 \mathrm{Mbps}$ & $30 \mathrm{FPS}$ & $180 \mathrm{p}, 270 \mathrm{p}, 360 \mathrm{p}, 450 \mathrm{p}, 540 \mathrm{p}, 630 \mathrm{p}, 720 \mathrm{p}$ & $2.0 \mathrm{Mbps}$ \\
\hline
\end{tabular}

Table 1: Summary of six video conferencing applications' characteristics.

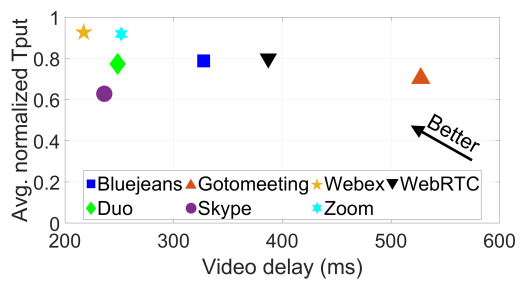

(a) Case 1: Packet loss variation $(0 \% \sim 20 \%)$

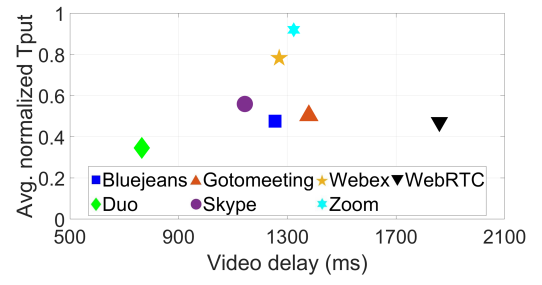

(b) Case 2: Delay variation $(+0 \mathrm{~ms} \sim+375 \mathrm{~ms})$

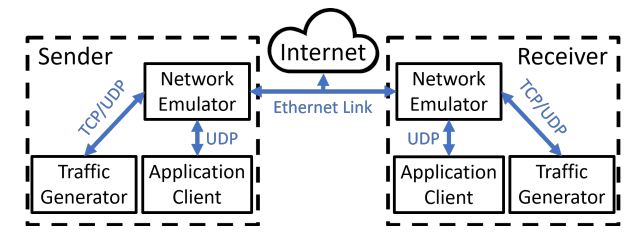

Figure 2: Testbed for measurement.

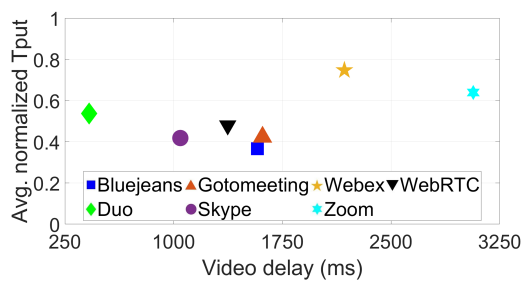

(c) Case 3: Throughput variation (500Kbps 5Mbps)

Figure 3: Performance comparison between six video conferencing applications and WebRTC.

delay. We observe that the sending rate of each application exceeds the maximum bitrate observed in the ideal scenario (Table 1). The increases come from loss recovery mechanisms such as forward error correction (FEC) and packet retransmission.

\subsection{Implication for Inferring the Rate Control}

We summarize key observations obtained from our extensive measurements, which provide guidelines to infer a proprietary rate control model of each application:

- The sender's rate control behaviors of all tested applications do not change based on the number of receivers, implying that inferring the rate control with a pair of clients is still applicable to multi-party scenarios.

- Most video conferencing applications take a similar principle to react to network dynamics from a macroscopic perspective. Specifically, (1) the video bitrate is linearly proportional to the available bandwidth, given no packet losses. (2) However, the gap between the sending rate in the transport layer and video bitrate at the application layer increases as packet losses increase due to its loss recovery mechanism such as FEC and retransmissions. (3) If an application's end-to-end delay increases over time, the video bitrate decreases to mitigate the congestion.

- Despite the similarity in all applications' macroscopic behavior, each application has a different degree of sensitivity against network dynamics such as packet loss and one-way delay variation.

To develop a generic framework for modeling popular video conferencing applications' rate control mechanisms, we first build a comprehensive model structure based on their common characteristics. Then, we scrutinize each application's distinct behavior with the shared model structure to find the best parameters that can approximate its unique responses for different network conditions.

\section{METHODOLOGY}

We describe our end-to-end testbed for real-time video streaming systems that emulates a time-varying network while measuring application-level video metrics. Also, we introduce key metrics and estimation methods for our evaluation.

\subsection{Experimental Setup}

Testbed setup. Figure 2 illustrates our testbed to emulate various network scenarios. Each client machine has an Intel 4-core $\mathrm{CPU}$ at $2.26 \mathrm{GHz}$ and 6GB RAM running Windows 10 , and each network emulator machine has an Intel 4 -core $\mathrm{CPU}$ at $1.8 \mathrm{GHz}$ and 32GB RAM running Ubuntu 16.04 with Linux kernel 4.15.0. The systems are connected via $1 \mathrm{Gbps}$ Ethernet with the network emulator, which performs traffic shaping through Linux TC command so that we can emulate a variety of network conditions such as available bandwidth, propagation delay, and packet loss rate. The network emulator is a gateway to the Internet. We inject either TCP or UDP flows into the network as background traffic via iPerf3 [13] using separate machines. We use CUBIC [20] for its TCP congestion control algorithm unless stated otherwise. We set up a pair of machines (up to two) to run each video call.

Video conferencing setup. For a video call, we keep playing a short video clip of standard TV news from CNN. The video mostly has head and shoulder movements, which is a typical content type for video conferencing. To capture the video, we use a Logitech HD C615 webcam which can capture up to 1080p [25]. It faces the screen displaying the TV news with a timestamp logging program in the corner of the screen. The timestamp represents the current time in a nanosecond to measure frame rate and streaming delay. Network conditions and real traces. We use not only synthetic network conditions to identify and verify the unique behaviors of each application, but also network traces collected in the literature $[6,29,32]$ to check the behavior of applications in realistic network environments. The network traces are a corpus of realworld throughput measured over three different networks: (i) FCC's consumer broadband performance in the U.S. [6], (ii) bandwidth measurements of $3 \mathrm{G}$ networks in Norway [29], and (iii) bandwidth measurements of 4G LTE networks in Belgium [32].

\subsection{Estimation of Key Metrics}

We need to estimate the following metrics for our measurement and model inference while running video conferencing applications.

Sending rate is the number of bits transmitted per second over the network, measured by using tcpdump. Based on the packet trace, 


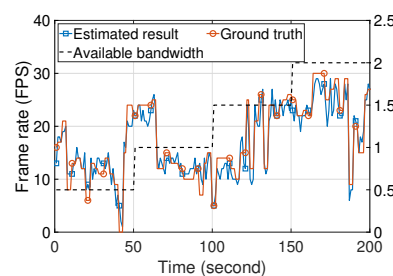

(a) Frame rate

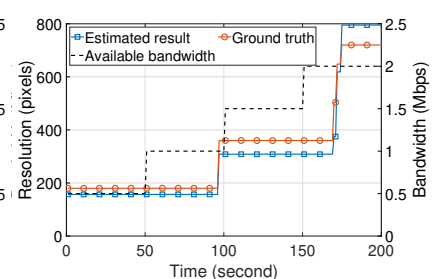

(b) Resolution
Figure 4: Accuracy of estimated metrics in Webex.

we extract the packets used for video conferencing and analyze them to calculate the sending rate (and resultant video bitrate).

Frame rate is the number of frames per second that appear on a screen. Specifically, we display a clock in nanoseconds at the corner of the video screen and capture the screen using the ScreenToGif program [27]. Then, we check the hash values of the captured screens to calculate how many times the screen changed per second. Resolution is the number of visible pixels in a video frame. We use an open-sourced tool called resdet [16] to obtain the estimated resolution. This tool identifies the original resolution of the image that may have been upscaled. We capture the recipient's screen every second and use it as the input image to this software, and take the output value as the estimated resolution.

Streaming delay is the time difference between the time when the video is generated at the sender and the time when the video is finally displayed at the receiver, which contains video capture, encoding, transmission, decoding, and rendering delays. To measure the streaming delay, we use the timestamp displayed at the corner of the screen. We record the sender's and the receiver's screens simultaneously and calculate the time difference by comparing the two screens' timestamps.

\subsection{Estimation Accuracy}

We evaluate the estimation accuracy using Webex for frame rates and resolutions since it explicitly provides the ground-truth values on the screen. We test the following scenario to see the adaptation behavior in Webex: we start with available bandwidth at $500 \mathrm{Kbps}$ and increase it by $500 \mathrm{Kbps}$ every 50 seconds. Then, we compare the results from our estimation method with the values obtained from Webex. Figures 4(a) and 4(b) show the estimation performance of our methods. As shown, we find that they can capture the varying trend achieved by Webex over time. The estimated results do not perfectly match the ground truth, but the errors are marginal. The similarity scores for frame rates and resolutions (defined in §5) show 0.94 and 0.92 , respectively. From this evaluation, we find that our estimation methods have a small error rate, so we apply them to all other applications hereafter.

\section{MODEL INFERENCE}

We first present research challenges and then model six representative applications' rate control algorithms and QoE-related metrics.

\subsection{Challenges}

As described in $\S 1$, a rate control algorithm is an essential part of today's video conferencing applications, which provides a control knob for both the source rate in the encoder and injection rate into the network. However, commercial applications' rate control algorithms are proprietary and not open to the public. To understand their behaviors with network dynamics, we need to infer their underlying algorithms, which involves addressing several practical challenges as follows:

- Challenge \#1: Many commercial video conferencing applications do not expose video quality information such as resolution, frame rate, and streaming latency ${ }^{1}$.

- Challenge \#2: All six video conferencing applications encrypt data packets such as video and voice traffic and signaling messages related to call setup for security.

- Challenge \#3: The real network is time-varying and highly diverse due to uncontrollable factors such as background traffic, the number of contending flows, and network dynamics.

\subsection{Our Approach}

To address the challenges mentioned above, we develop a novel method to obtain video rate and quality information by exploiting open-source analysis tools (as described in §3.2) so that we can get the necessary information at high accuracy. We then build a generic model that can capture all applications' common behaviors. In particular, we adopt WebRTC's GCC algorithm framework [21, 22], which is generally enough to implement any specific rate adaption algorithm used in an individual video-conferencing application. The model captured for each application is then implemented in the WebRTC framework for validation in $§ 5$.

\subsection{Rate Control Framework}

WebRTC's GCC algorithm consists of two controllers: (i) delaybased control at the receiver and (ii) loss-based control at the sender, shown in Figure 5.

At the receiver, the delay controller estimates the delay variation (denoted by $\Delta D)^{2}$ from the one-way delay measurements between two consecutive video frames. The delay-based controller uses such delay variation to adapt to the dynamic network condition. Specifically, it decides whether to increase, decrease, or hold the target bitrate $\left(A_{d}\right)$ by comparing the one-way delay variation with the threshold value $\left(D_{T h}\right)$, as follows:

$$
A_{d}\left(t_{i}\right)= \begin{cases}A_{d}\left(t_{i-1}\right), & \text { if } \Delta D<-D_{T h} \\ \beta R\left(t_{i}\right), & \text { if } \Delta D>D_{T h} \\ \alpha A_{d}\left(t_{i-1}\right), & \text { otherwise }\end{cases}
$$

where $t_{i}$ is the time when the $i$ th video frame is received at the receiver, $\alpha$ and $\beta$ are key factors for "increase" and "decrease" operations, and $R\left(t_{i}\right)$ is the receiving rate during the last $500 \mathrm{~ms}$.

On the other hand, at the sender, the loss-based controller computes the target sending rate based on the fraction of lost RTP (Real-time Transport Protocol) packets whenever it receives RTCP (RTP Control Protocol) feedback from the receiver. Similarly to the delay-based control, it relies on two key parameters, $\alpha$ and $\beta$, to represent both "increase" and "decrease" states. Finally, the lossbased control model computes the target bitrate $\left(A_{l}\right)$ as:

\footnotetext{
${ }^{1}$ Among six applications, Webex and Zoom provide such video quality information, and Bluejeans provides resolution only.

${ }^{2}$ Typically, queueing at the bottleneck link can incur such delay variation, given the end-to-end routing path is fixed, often called bufferbloat [17].
} 


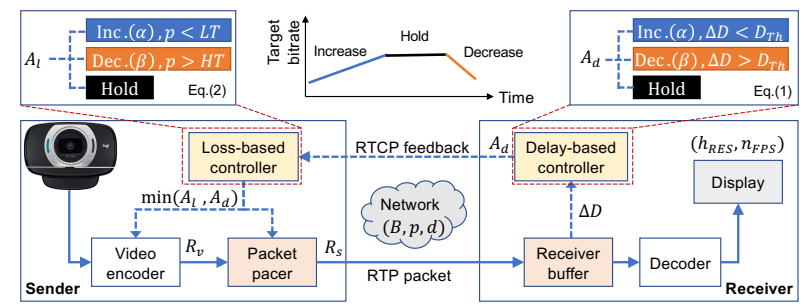

Figure 5: Diagram of the end-to-end rate control architecture for model inference: For the loss-based control, two adaptation parameters $\alpha$ and $\beta$ and two thresholds $L T$ and $H T$ are used, while for the delay-based control, two adaptation parameters $\alpha$ and $\beta$ and one threshold $D_{T h}$ are used.

$$
A_{l}\left(t_{k}\right)= \begin{cases}\alpha A_{l}\left(t_{k-1}\right), & \text { if } p<L T \\ \beta\left(A_{l}\left(t_{k-1}\right)\right), & \text { if } p>H T \\ A_{l}\left(t_{k-1}\right), & \text { otherwise }\end{cases}
$$

where $t_{k}$ denotes the time when the $k$ th feedback is received at the sender and $p$ is the estimated packet loss rate. Note that the final target bitrate for the video encoder is taken by the minimum of the values estimated in the two controllers above.

We emphasize that the structure above can accommodate various adaptation patterns of video conferencing applications by changing a combination of key parameters such as $\alpha, \beta$, and threshold parameters (i.e., $L T, H T, D_{T h}$ ). Figure 5 shows the mapping of key parameters in the rate control architecture.

\subsection{Modeling Rate Control Algorithm}

Our goal is to find key parameters that closely follow each application's unique rate control behavior.

Delay-based rate controller. The minimum threshold $\left(D_{T h}\right)$ represents an application's sensitivity to delay variation. We search this threshold using a binary search. Specifically, we start from a sufficiently large threshold value (e.g., 400ms) and search the threshold for each application, which lies in $(0,400 \mathrm{~ms}]$. Since the application would have a significant throughput difference before and after the threshold, we finish the search if the throughput difference before and after the threshold is substantial (in our case, $>15 \%$ of throughput difference). Table 2 shows the minimum threshold $\left(D_{T h}\right)$ for six applications. We can see that Webex and Zoom have larger threshold values, indicating that they prefer to maintain the current rate by not reacting quickly to delay variations.

Next, we infer the adaptation parameters deciding how much to increase $(\alpha)$ and how much to reduce $(\beta)$ its sending rate based on delay variations. This process is relatively straightforward as long as we already know the delay threshold $\left(D_{T h}\right)$ of each application. We compute the throughput slope before and after this threshold for many runs over the networks of different RTTs. The inferred parameters for six applications are summarized in Table 2. Zoom has larger $\alpha$ and $\beta$ values than the other applications, indicating that Zoom increases its sending rate more aggressively if the network condition is good while it reduces less if the network suffers from severe delay increases. According to the inferred parameters, Zoom has the largest $D_{T h}, \alpha$ and $\beta$ values, which explains why it has ranked highly for its streaming quality [31, 34].

\begin{tabular}{|c|c|c|c|c|c|c|c|}
\hline \multirow{2}{*}{ Application } & \multicolumn{3}{|c|}{ Delay-based control } & \multicolumn{4}{c|}{ Loss-based control } \\
\cline { 2 - 8 } & $\begin{array}{c}\text { Thres. } \\
\left(D_{T h}\right)\end{array}$ & $\begin{array}{c}\text { Inc. } \\
\text { fac. }(\alpha)\end{array}$ & $\begin{array}{c}\text { Dec. } \\
\text { fac. }(\beta)\end{array}$ & $\begin{array}{c}\text { Low thres. } \\
(L T)\end{array}$ & $\begin{array}{c}\text { High thres. } \\
(H T)\end{array}$ & $\begin{array}{c}\text { Inc. } \\
\text { fac. }(\alpha)\end{array}$ & $\begin{array}{c}\text { Dec. } \\
\text { fac. }(\beta)\end{array}$ \\
\hline \hline Bluejeans & $60 \mathrm{~ms}$ & 1.10 & 0.90 & $6 \%$ & $15 \%$ & 1.05 & 0.90 \\
\hline Duo & $10 \mathrm{~ms}$ & 1.05 & 0.85 & $2 \%$ & $8 \%$ & 1.25 & 0.95 \\
\hline Gotomeeting & $10 \mathrm{~ms}$ & 1.05 & 0.80 & $20 \%$ & $33 \%$ & 1.15 & 0.88 \\
\hline Skype & $60 \mathrm{~ms}$ & 1.05 & 0.90 & $10 \%$ & $10 \%$ & 1.09 & 0.95 \\
\hline Webex & $160 \mathrm{~ms}$ & 1.04 & 0.90 & $10 \%$ & $34 \%$ & 1.04 & 0.85 \\
\hline Zoom & $360 \mathrm{~ms}$ & 1.20 & 0.90 & $11 \%$ & $45 \%$ & 1.20 & 0.90 \\
\hline
\end{tabular}

Table 2: Parameters for rate control models, Eqs. (1) and (2).

\begin{tabular}{|c|c|c|c|c|c|c|c|c|c|}
\hline \multirow{2}{*}{ Application } & \multicolumn{2}{|c|}{ FEC ratio } & \multicolumn{2}{|c|}{ Sending rate } & \multicolumn{2}{c|}{ Frame rate } & \multicolumn{2}{c|}{ Resolution } \\
\cline { 2 - 10 } & $\omega$ & $\psi$ & $\gamma$ & $\mu$ & $c$ & $\rho_{F}$ & $\epsilon_{F}$ & $\rho_{R}$ & $\epsilon_{R}$ \\
\hline \hline Bluejeans & 4.16 & 0.014 & 0.81 & -2.54 & 275 & 0.013 & 6.912 & 0.496 & 156.78 \\
\hline Duo & 1.11 & 0.014 & 0.72 & -1.91 & 120 & 0.056 & 6.384 & 0.507 & 203.50 \\
\hline Gotomeeting & 1.11 & 0.011 & 0.78 & -1.36 & 230 & 0.113 & 0.645 & 0.560 & 114.07 \\
\hline Skype & 0.41 & 0.000 & 0.89 & -1.34 & 570 & 0.041 & 0.456 & 0.431 & 195.89 \\
\hline Webex & 7.52 & 0.015 & 0.89 & -1.71 & N/A & 0.026 & 5.162 & 0.395 & 229.30 \\
\hline Zoom & 4.42 & 0.032 & 0.88 & -1.57 & 600 & 0.015 & 4.500 & 0.344 & 209.51 \\
\hline
\end{tabular}

Table 3: Parameters for video QoE models, Eqs. (3) (7). The sending rate consists of the video bitrate and FEC portion.

Loss-based rate controller. Similar to the delay threshold, the loss thresholds represent an application's sensitivity to packet losses. The application increases its rate if the observed loss rate is less than a lower threshold $(L T)$ and decreases if the loss rate is greater than a higher threshold $(H T)$. Otherwise, the application maintains the current sending rate. Again, we search these two thresholds for each application using a binary search from a sufficiently large loss rate (e.g., 50\%). Table 2 shows the two thresholds ( $L T$ and HT) and rate adaption parameters $(\alpha$ and $\beta$ ) for each application using the same methodology used for the delay-based rate controller. Google's Duo is most sensitive to packet losses and is expected to share the bandwidth with other streaming or TCP flows fairly well by reducing its sending rate for other flows. Again, Zoom has a larger gap between $L T$ and $H T$, indicating its design strategy of maintaining the current rate as much as possible.

\subsection{Modeling QoE-related Metrics}

Now, we present our analytical model for QoE-related parameters such as FEC ratio, sending rate, video rate, frame rate, and resolution of six applications. These models allow us to predict the performance of the applications in a steady state.

FEC ratio. To infer the amount of FEC packets of each application under different packet loss rates, we presume that the video bitrate remains the same if the frame rate and resolution for video traffic are kept at the same level. Actually, we observe this pattern in our extensive measurements $(\S 2)$. We measure the sending rate, frame rate, and resolution of all the applications one by one under mild packet losses ranging between 0 and $10 \%$ in the network. We find that the amount of FEC packets increases linearly to the injected packet loss rate. To express the relationship between the FEC portion and packet loss rate $p$, we use linear regression in modeling the FEC ratio (denoted by $r_{F E C}$ ) as follows:

$$
r_{F E C}(p)=\omega p+\psi
$$

where $\omega$ and $\psi$ are constants derived from the curve fitting. The values for six applications are summarized in Table 3.

Sending rate. From the bandwidth measurements in $\S 2$, we confirmed that all applications adjust the sending rate following the available bandwidth with some margin until the maximum rate is reached. Hence, we use linear regression to build the sending rate model to capture the increase in proportion to the bandwidth. 
Furthermore, we confirmed that the actual sending rate could exceed the maximum rate due to FEC in the presence of packet losses. By taking the minimum from the two values, we have a complete sending rate model. On the other hand, we observed that all applications reduce the sending rate significantly to a low level when the packet loss rate goes beyond the high threshold (i.e., $H T$ ). Let $R_{S}$ be the actual sending rate and $R_{M}$ be the maximum bitrate (listed in Table 1), then we have a sending rate model as follows:

$$
R_{s}(B, p)= \begin{cases}\min \left(\gamma B+\mu, R_{M}(1+\psi+\omega p)\right), & \text { if } p \leq H T \\ c, & \text { otherwise }\end{cases}
$$

where $B$ is the network bandwidth, $c$ denotes the lowest observed sending rate, and $\gamma, \mu, \psi$, and $\omega$ are model parameters summarized in Table 3. HT represents the high threshold listed in Table 2. Note that unlike other applications, the sending rate of Webex does not converge to the single rate in the network conditions whose packet loss rate exceeds HT. Also, if the packet loss exceeds $50 \%$, the video call is disconnected. For this reason, we mark Webex's case as N/A. Video bitrate. We reconstructed the rate control algorithm of each application in $\S 4.4$. As a result, the target bitrate obtained from the rate control mechanism would be closely related to the video rate since the target bitrate is used as an input value for the video encoder. In other words, the target bitrate and the (resultant) video rate should be the same (or similar) on a roughly one-second timescale [15]. In fact, the rate control model describes how the application adapts to network dynamics, while the video bitrate model allows us to indicate the bitrate in the steady-state. Since the sending traffic mainly consists of video data and the FEC portion, we can build the video bitrate model by subtracting the FEC portion from the estimated sending rate. Let $R_{v}$ be the video bitrate (in Kbps) and $R_{M}$ be the maximum bitrate, and then we can express the video bitrate model as follows:

$$
R_{v}(B, p)= \begin{cases}\min \left((1-\psi-\omega p)(\gamma B+\mu), R_{M}\right), & \text { if } p \leq H T \\ (1-\psi-\omega p) c, & \text { otherwise }\end{cases}
$$

where all symbols are the same as those in the sending rate model. Frame rate. We confirmed that the application's frame rate changes as the video bitrate adapts to the varying bandwidth (in §2). Thus, we investigate the precise relationship between the video bitrate and frame rate. We observe that overall, all applications increase the frame rate proportional to the video bitrate until the maximum frame rate is reached, from which, we propose the frame rate model as a linear function with respect to the achieved video bitrate bounded by the maximum frame rate:

$$
n_{F P S}\left(R_{v}\right)=\min \left(\rho_{F} R_{v}+\epsilon_{F}, n_{F P S}^{\max }\right)
$$

where $n_{F P S}^{\max }$ is the maximum frame rate listed in Table $1, \rho_{F}$ and $\epsilon_{F}$ are model parameters summarized in Table 3.

Video resolution. In $\S 2$, we show that the video resolution is closely related to the video bitrate. We deduce that all applications determine the resolution depending on the current video bitrate. Similar to the frame rate model, we propose the resolution model in terms of the video bitrate as follows:

$$
h_{R E S}\left(R_{v}\right)=\min \left(\rho_{R} R_{v}+\epsilon_{R}, h_{R E S}^{\max }\right)
$$

where the inferred model parameters $\rho_{R}$ and $\epsilon_{R}$ are in Table 3, and $h_{R E S}^{\max }$ denotes the maximum observed resolution found in Table 1 . Note that our resolution model is a linear function while the resolutions shown in Table 1 are discrete.

From the linear regression models, we obtain $R^{2}=0.927$ on average, indicating that our models fit the data well.

\section{MODEL VALIDATION}

Now, we evaluate the accuracy of the proposed models. We implement them in WebRTC, and compare the resultant behavior with the actual behavior of each applications under the same conditions.

To measure the accuracy between two obtained results, we use the similarity score, defined as $1-d\left(\frac{x_{\text {orig }}-x_{\min }}{x_{\max }-x_{\min }}, \frac{x_{\text {model }}-x_{\min }}{x_{\max }-x_{\min }}\right)$, where $x_{\min }$ and $x_{\max }$ are the minimum and maximum values observed during the entire video transmission, $x_{\text {model }}$ and $x_{\text {orig }}$ are vectors of the measured parameter (e.g., bitrate), and $d(\cdot)$ computes the Euclidean distance between the resulting vectors. To scale in $[0,1]$, we use the min-max normalization of the two measurements [33]. For intuitive interpretation, we express the similarity score by subtracting the normalized distance from 1 , so the score of 1 means a perfect match, while the score of 0 means a mismatch.

\subsection{Choice of WebRTC Platform}

WebRTC is a de facto standard for live video streaming that has been incorporated into major web browsers. We choose this platform due to the following reasons: First, WebRTC allows flexible reimplementation of the video control algorithms and has been used in the state-of-the-art transport and video application researches, such as Salsify [15], Concerto [39] and OnRL [37], etc. Second, WebRTC's open-source reference implementation provides an independent modular architecture including two major components: a transport protocol and a video codec. This architectural separation is shared by many commercial applications such as Skype, FaceTime, and Hangouts [15]. Third, while commercial applications have their own implementation details, which are likely different from WebRTC, our measurements confirmed that their rate control algorithms share three fundamental operations: increase, decrease, and maintain the rate under specific conditions, which can be easily implemented in WebRTC.

\subsection{Rate Control Algorithm Model}

Delay and loss-based control validation. We verify whether our proposed rate control model can follow the actual behavior of six applications. Based on the inferred threshold values (shown in Table 2), we consider two artificial scenarios to emulate delay and packet loss dynamics separately. In particular, for the dynamic delay experiment, we vary the additional propagation delay every minute in the network while fixing the available bandwidth and packet loss rate with $10 \mathrm{Mbps}$ and $0 \%$, respectively. This setup can cover the range of delays in Table 2 . For the dynamic packet loss experiment, we change the packet loss rate every minute, ranging from 0 to $20 \%$, while maintaining the bandwidth and base RTT with $10 \mathrm{Mbps}$ and $50 \mathrm{~ms}$, respectively. Using these scenarios, we compare the sending rate achieved by the proposed models and the target applications. We report the average and standard deviation. 


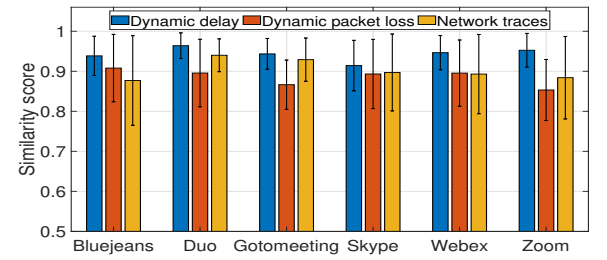

Figure 6: Similarity scores of each application's rate control (inferred vs. real). The error bars plot the $\mathrm{min} / \mathrm{max}$ values.

Figure 6 shows the resultant similarity scores in the achieved sending rate between our model and actual measurement. The blue bar represents the similarity score obtained from the delay experiment, and the red bar is the score from the loss experiment. We find that across all applications the similarity scores are 0.943 and 0.885 on average, respectively, implying that the inferred models can fairly capture the behavior of the commercial applications.

Evaluation using real network traces. We also validate our inferred models using trace-driven emulation. For this, we exploit real-world network traces consisting of 5 most unstable, 5 most steady, and 5 random traces from the collection of the U.S. [6], Norway [29], and Belgium [32] dataset. We use a total of 45 network traces characterized by throughput variation ranging from $102.45 \mathrm{Kbps}$ to $14.61 \mathrm{Mbps}$ and an average of $2.77 \mathrm{Mbps}$.

In Figure 6, the yellow bar shows the harmonic mean of similarity scores evaluated across all network traces. Overall, we have a similarity score of 0.903 on average, indicating that the proposed models faithfully follow the sending rate of the original applications.

\subsection{QoE-related Metrics Model}

FEC ratio model validation. Our hypothesis is that the sending rate consists of FEC data rate and video data rate, and the video rate is kept constant as long as the frame rate and resolution are maintained. Thus, we estimate the FEC data rate by subtracting the expected video rate from the total sending rate measured, where the expected video rate is equal to the sending rate (other than header overhead) at $0 \%$ packet loss. By comparing the estimated FEC portion with the ground-truth obtained from WebRTC, we verify that our estimation method on the FEC portion works well in WebRTC. We compare our FEC ratio models with the measurements obtained by running six applications under mild packet loss rates, ranging from $0.5 \%$ to $5 \%$. The y-axis of Figure 7 (a) shows similarity scores between the inferred models and original behaviors achieved by six applications. The similarity score of the FEC ratio model is 0.863 on average, indicating the FEC ratio model is fairly reliable. Sending rate, frame rate, and resolution models validation. We design the experiments where each application flow shares a bottleneck link with background UDP flows. The UDP flows with constant sending rates from 0 to $2800 \mathrm{Kbps}$ are injected into the network whose bandwidth and delay are set to $3000 \mathrm{Kbps}$ and $50 \mathrm{~ms}$, respectively. After all target applications enter the steady state (e.g., 60 seconds from the start), we collect the traces on sending rate, frame rate, and resolution. Also, our models are used to predict the sending rate, frame rate, and resolution, given that the available bandwidth is $3000 \mathrm{Kbps}$ minus background UDP flows' rate. Figure 7 shows the similarity scores in terms of the sending rate, frame rate, and resolution. The $\mathrm{x}$-axis of Figure 7(a) represents the result of

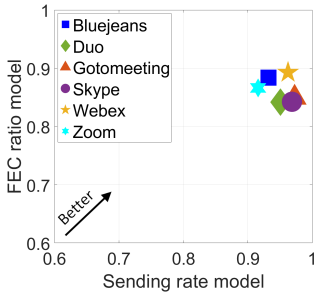

(a) Sending rate and FEC ratio

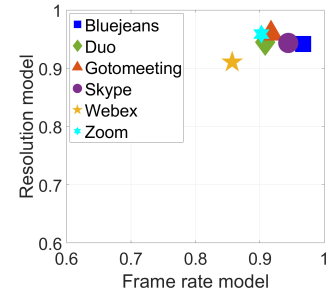

(b) Frame rate and resolution
Figure 7: Validation for QoE-related metric modeling.

sending rate models. The $\mathrm{x}$-axis and $\mathrm{y}$-axis of Figure $7(\mathrm{~b})$ are the results of frame rate and resolution models, respectively. The average similarity scores for the sending rate, frame rate, and resolution inference models are $0.950,0.924$, and 0.943 , respectively, indicating that our model predictions match the corresponding behaviors for all applications.

\section{MODEL-DRIVEN ANALYSIS}

\subsection{Cross-Application Contention}

We evaluate how each application behaves when competing with each other among six applications, which can be analyzed using our rate control models.

Setup: We consider two concurrent application flows over a $2 \mathrm{Mbps}$ link. We set the queue size of the bottleneck link with 100 packets and a round-trip delay of $50 \mathrm{~ms}$. We use two pairs of sender and receiver machines to generate separate video calls. After the first flow enters the steady-state, we enable the second flow and measure the throughput for 120 seconds.

Results: Figure 8 shows the average bitrate achieved by two flows in all combinations, where the bar on the left indicates the first flow's bitrate and the bar on the right represents the second flow's bitrate. We observe important observations from this experiment: First, all applications fairly share the bottleneck link when competing with their own applications. Second, when competing with other applications, each application shows very different behaviors depending on which one to compete with and which one occupies first. For example, when contending with Zoom, most applications yield much less performance than their fair share. Also, in the Skype-Duo combination, when Duo arrives later than Skype, Duo gets very little bandwidth. However, when Skype comes later than Duo, Skype manages to get a fair share. Our rate control models can clearly explain this. As shown in Table 2, Zoom uses the largest delay threshold compared to others, so it tries to maintain the current rate as much as possible even with the increased delay caused by the competing flows, while Duo and Gotomeeting use a relatively small threshold, providing more room for competing flows to increase their rates.

\subsection{Coexistence with TCP Flows}

We investigate the performance of two applications, Duo and $\mathrm{Zoom}^{3}$ in the presence of competing TCP flows. The purpose is to understand their behavior in terms of delay and loss-based control mechanisms when the video call flow contends with a TCP flow.

${ }^{3}$ Duo is the most conservative, while Zoom is the most aggressive, according to their rate control parameters shown in Table 2 . 


\begin{tabular}{|c|c|c|c|c|c|c|}
\hline$F 1 \quad F 2$ & Bluejeans & Duo & Gotomeeting & Skype & Webex & zoom \\
\hline Bluejeans & En & ne & En & EE & - & $=$ \\
\hline Duo & $-\square$ & E & -1 & E & a & -1 \\
\hline Gotomeeting & 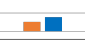 & I & n日 & n & m & $=$ \\
\hline Skype & En & 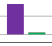 & Q & E & m & - \\
\hline Webex & $=$ & 1 & - & - & $m$ & n \\
\hline Zoom & n & E & L & E & D & n \\
\hline
\end{tabular}

Figure 8: Experiment results for cross-app contention: the left bar is 1st flow (F1) and the right bar is 2nd flow (F2). The color of each bar indicates a specific application.

Setup: We set the bottleneck bandwidth to $3 \mathrm{Mbps}$ along with the buffer of 100 packets and the base RTT of 50ms between two endpoints. We first run a video call and then inject a TCP flow with two congestion control algorithms, CUBIC [20] and BBR (v1) [3], where CUBIC adjusts the sending rate by increasing the window until packet loss occurs, while BBR adjusts the sending rate using pacing technique based on the estimated BDP irrespective of packet loss. Three scenarios are tested with different packet losses of $0-$ $10 \%$ to see the impact of packet loss.

Results: Figure 9 depicts the throughput dynamics achieved by the video flow and TCP flow. Interestingly, we observe a clear gap between the two video applications. When contending with a TCP CUBIC flow, Duo could utilize the remaining bandwidth under 5\% packet loss rates only because the TCP CUBIC flow had difficulty in increasing its sending rate due to its sensitivity to severe losses. Under $0 \%$ loss rates, Duo reduces its bitrate due to the increased delay by the TCP CUBIC flow. Under $10 \%$ loss rates, Duo also reduces the bitrate due to its loss-based control. However, when contending with TCP BBR, Duo gets starved by the delay-based control, irrespective of packet loss rates.

Next, we analyze Zoom's case. When competing with a TCP CUBIC flow, Zoom loses its throughput to the TCP CUBIC flow under no packet loss, as the TCP CUBIC flow fills up the buffer and increases the RTT (up to 791ms), making Zoom's delay-based control reduces the bitrate. However, CUBIC cannot increase its window sufficiently when packet loss happens, allowing Zoom to restore its bitrate to the previous state. When contending with TCP $\mathrm{BBR}$, Zoom predominates over BBR regardless of the packet loss rates because BBR does not increase delay significantly due to its low latency feature, thus letting Zoom operate vigorously.

Our analysis demonstrates that Zoom performs aggressively compared to other applications, which can explain why many users are satisfied with Zoom's video call qualities, as reported in several technical blogs, e.g., [31, 34]. However, making a rate control algorithm more aggressive for its own benefits could hurt the performance of other applications.

\section{RELATED WORK}

Understanding commercial platforms. Since the seminal work on Skype [1], many papers have attempted to understand the architecture of Skype $[2,19]$. Cicco et al. experimentally revealed that Skype used various sending rates to match the available bandwidth of network [11] and provided a mathematical model [10]. Moreover, they investigated Skype's congestion control to figure out how to

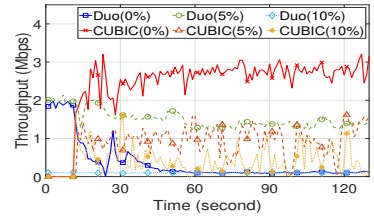

(a) Duo vs. CUBIC

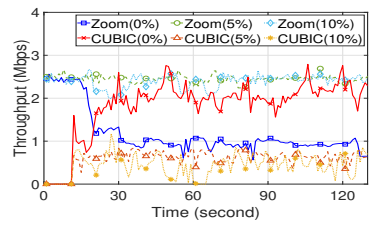

(c) Zoom vs. CUBIC

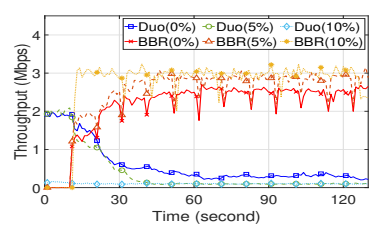

(b) Duo vs. BBR

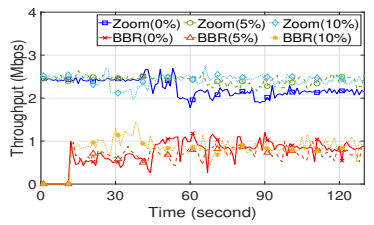

(d) Zoom vs. BBR
Figure 9: Throughput dynamics in the coexistence scenario of a video and a TCP flows with varying packet loss.

adapt to the network bandwidth [12]. Zhang et al. [38] conducted an extensive measurement of Skype under various conditions and proposed models for its rate control, FEC, and video quality. Xu et al. [36] compared Google+, iChat, and Skype and unveiled the architecture of these applications along with performance evaluation. Fouladi et al. [15] proposed Salsify, a unique architecture for a realtime video that tightly integrates a video codec and transport-layer control to respond quickly to changing network conditions.

Understanding WebRTC. Since its debut [26] in 2011, several papers evaluated the performance of WebRTC's congestion control. Cicco et al. [8] assessed the GCC algorithm using a controlled testbed and found the starvation issue when competing with TCP [9]. Carlucci et al. [4] analyzed GCC's performance using a queueing delay gradient to infer congestion. They showed that GCC adapts to the link capacity and provides intra-protocol and interprotocol fairness. More realistic and massive evaluation [22] showed that WebRTC suffered from poor performance over wireless networks due to bursty losses. To improve the live video system, many works [15, 23, 24, 35, 37, 39] exploit WebRTC as a reference.

\section{CONCLUSION}

In this paper, we conducted a measurement-based comparison study of six popular video conferencing applications. Our evaluation indicated that they exhibit common behavior from a macroscopic perspective, motivating us to model their rate control algorithms using a unified architecture. We exploited WebRTC's modular structure to infer key parameters that can capture each application's unique features under a range of network dynamics. More importantly, these inferred models can be used to acquire an in-depth understanding of the proprietary video conferencing applications' inefficiency and vulnerability, especially when contending with other flows. We believe our effort will encourage further study on commercial video conferencing applications.

\section{ACKNOWLEDGMENTS}

We thank Ibrahim Ayad, Sangeeta Ramakrishnan and Xiaoqing Zhu for their feedback on the earlier work of this study. This research was supported in part by Cisco Systems (Grant 1368170) and the IITP grant funded by the Korea government (MSIT) (2018-0-00693). 


\section{REFERENCES}

[1] Salman A. Baset and Henning Schulzrinne. An analysis of the skype peer-to-peer internet telephony protocol. arXiv preprint cs/0412017, 2004.

[2] Dario Bonfiglio, Marco Mellia, Michela Meo, Nicolo Ritacca, and Dario Rossi. Tracking down skype traffic. In Proceedings of IEEE INFOCOM, 2008.

[3] Neal Cardwell, Yuchung Cheng, C Stephen Gunn, Soheil Hassas Yeganeh, and Van Jacobson. BBR: Congestion-Based Congestion Control. ACM Queue, 14(5):50, 2016.

[4] Gaetano Carlucci, Luca De Cicco, Stefan Holmer, and Saverio Mascolo. Analysis and design of the google congestion control for web real-time communication (WebRTC). In Proceedings of ACM MMSys, 2016.

[5] Cisco. Bandwidth Planning in your Cisco Webex Meetings Environment White Paper. https://www.cisco.com/c/en/us/products/collateral/conferencing/webexmeetings/white_paper_c11-691351.html, 2018.

[6] Federal Communications Commission. Validated data - measuring fixed broadband - ninth report. https://www.fcc.gov/reports-research/reports/.

[7] Dataset and tools used for the paper, titled "Demystifying Commercial Video Conferencing Applications", ACM Multimedia 2021. https://bit.ly/3rBpNkF, 2021.

[8] Luca De Cicco, Gaetano Carlucci, and Saverio Mascolo. Experimental investigation of the google congestion control for real-time flows. In Proceedings of ACM SIGCOMM workshop on Future human-centric multimedia networking, 2013.

[9] Luca De Cicco, Gaetano Carlucci, and Saverio Mascolo. Understanding the dynamic behaviour of the google congestion control for rtcweb. In Proceedings of 20th International Packet Video Workshop. IEEE, 2013.

[10] Luca De Cicco and Saverio Mascolo. A mathematical model of the Skype VoIP congestion control algorithm. IEEE Transactions on Automatic Control, 55(3):790$795,2010$.

[11] Luca De Cicco, Saverio Mascolo, and Vittorio Palmisano. An experimental investigation of the congestion control used by Skype VoIP. In Proceedings of International Conference on Wired/Wireless Internet Communications. Springer, 2007.

[12] Luca De Cicco, Saverio Mascolo, and Vittorio Palmisano. Skype video congestion control: An experimental investigation. Computer Networks, 55(3):558-571, 2011.

[13] Jon Dugan, Seth Elliott, Bruce A. Mah, Jeff Poskanzer, and Kaustubh Prabhu iPerf - The ultimate speed test tool for TCP, UDP and SCTP. https://iperf.fr/iperfdownload.php/.

[14] M. Fasciani, T. Eagle, and A. Preset. Magic quadrant for meeting solutions. Technical report, Gartner, 2018.

[15] Sadjad Fouladi, John Emmons, Emre Orbay, Catherine Wu, Riad S. Wahby, and Keith Winstein. Salsify: Low-Latency Network Video through Tighter Integration between a Video Codec and a Transport Protocol. In Proceedings of USENIX NSDI, 2018.

[16] Ruslan Gafurov and Jonti Olds. Resdet - detect source resolution of upscaled images. https://github.com/0x09/resdet/.

[17] Jim Gettys and Kathleen Nichols. Bufferbloat: Dark Buffers in the Internet: Networks without Effective AQM May Again Be Vulnerable to Congestion Collapse. ACM Queue, 9(11):40-54, November 2011.

[18] Google. Real-time communication for the web. https://webrtc.org, 2020.

[19] Saikat Guha and Neil Daswani. An experimental study of the skype peer-to-peer voip system. Technical report, Cornell University, 2005.

[20] Sangtae Ha, Injong Rhee, and Lisong Xu. CUBIC: a New TCP-friendly High-speed TCP Variant. ACM SIGOPS Operating Systems Review, 42:64-74, July 2008.

[21] Stefan Holmer, Henrik Lundin, Gaetano Carlucci, Luca De Cicco, and Saverio Mascolo. A google congestion control algorithm for real-time communication, draft-ietf-rmcat-gcc-02, 2016.

[22] Bart Jansen, Timothy Goodwin, Varun Gupta, Fernando Kuipers, and Gil Zussman. Performance evaluation of webrtc-based video conferencing. ACM SIGMETRICS Performance Evaluation Review, 45(3):56-68, 2018.

[23] Jaehong Kim, Youngmok Jung, Hyunho Yeo, Juncheol Ye, and Dongsu Han. Neural-enhanced live streaming: Improving live video ingest via online learning. In Proceedings of ACM SIGCOMM, 2020.

[24] Jinsung Lee, Sungyong Lee, Jongyun Lee, Sandesh Dhawaskar Sathyanarayana, Hyoyoung Lim, Jihoon Lee, Xiaoqing Zhu, Sangeeta Ramakrishnan, Dirk Grunwald, Kyunghan Lee, and Sangtae Ha. PERCEIVE: Deep Learning-Based Cellular Uplink Prediction Using Real-Time Scheduling Patterns. In Proceedings of ACM MobiSys, 2020.

[25] Logitech. Logitech c615 portable hd 1080p webcam with autofocus. https: //www.logitech.com/en-us/product/hd-webcam-c615/.

[26] Salvatore Loreto and Simon Pietro Romano. Real-time communications in the web: Issues, achievements, and ongoing standardization efforts. IEEE Internet Computing, 16(5):68-73, 2012.

[27] Nicke Manarin. Screentogif: screen, webcam and sketchboard recorder with an integrated editor. https://www.screentogif.com/.

[28] Microsoft. How much bandwidth does Skype need? https://support.skype.com/ en/faq/FA1417/how-much-bandwidth-does-skype-need, 2020.

[29] Haakon Riiser, Paul Vigmostad, Carsten Griwodz, and Pål Halvorsen. Commute path bandwidth traces from $3 \mathrm{G}$ networks: analysis and applications. In Proceedings of ACM MMSys, 2013.

[30] Transparency Market Research. Video conferencing market. https:// www.transparencymarketresearch.com/video-conferencing-market.html, 2020.

[31] User Interviews. The Best Video Conferencing Tools for Remote User Testing. https://www.userinterviews.com/blog/the-best-video-conferencing-toolsfor-remote-user-testing, 2020.

[32] Jeroen Van Der Hooft, Stefano Petrangeli, Tim Wauters, Rafael Huysegems, Patrice Rondao Alface, Tom Bostoen, and Filip De Turck. HTTP/2-based adaptive streaming of HEVC video over 4G/LTE networks. IEEE Communications Letters, 20(11):2177-2180, 2016.

[33] Wikipedia. Feature scaling. https://en.wikipedia.org/wiki/Feature_scaling.

[34] Wirecutter. The Best Videoconferencing Service. https://www.nytimes.com/ wirecutter/reviews/best-video-conferencing-service/, 2020.

[35] Xiufeng Xie and Xinyu Zhang. POI360: Panoramic Mobile Video Telephony over LTE Cellular Networks. In Proceedings of ACM CoNEXT, 2017.

[36] Yang Xu, Chenguang Yu, Jingjiang Li, and Yong Liu. Video Telephony for EndConsumers: Measurement Study of Google+, iChat, and Skype. IEEE/ACM Transactions on Networking, 22(3):826-839, 2014.

[37] Huanhuan Zhang, Anfu Zhou, Jiamin Lu, Ruoxuan Ma, Yuhan Hu, Cong Li, Xinyu Zhang, Huadong Ma, and Xiaojiang Chen. OnRL: Improving Mobile Video Telephony via Online Reinforcement Learning. In Proceedings of ACM MobiCom, 2020.

[38] Xinggong Zhang, Yang Xu, Hao Hu, Yong Liu, Zongming Guo, and Yao Wang. Profiling skype video calls: Rate control and video quality. In Proceedings IEEE INFOCOM, 2012.

[39] Anfu Zhou, Huanhuan Zhang, Guangyuan Su, Leilei Wu, Ruoxuan Ma, Zhen Meng, Xinyu Zhang, Xiufeng Xie, Huadong Ma, and Xiaojiang Chen. Learning to Coordinate Video Codec with Transport Protocol for Mobile Video Telephony. In Proceedings of ACM MobiCom, 2019.

[40] Zoom. Bandwidth requirements. https://support.zoom.us/hc/en-us/articles/ 201362023-System-requirements-for-Windows-macOS-and-Linux, 2020. 